\title{
Enhancing cultural competence in mental health settings: from undergraduate training to continuing education
}

\author{
Shoba Nayar \\ Doctoral Candidate, Department of Occupational Science and Therapy, Auckland University of \\ Technology University, New Zealand \\ Samson Tse \\ Associate Professor, Department of Social Work and Social Administration, The University of Hong Kong \\ Amritha Sobrun-Maharaj \\ Director of the Centre for Asian Health Research and Evaluation, School of Population Health, \\ The University of Auckland, New Zealand
}

\begin{abstract}
New Zealand is a country of many cultures and ethnicities. With a growing Asian community, cultural capability of the mental health workforce is essential to ensure that Asians have equity in access to appropriate and high quality services. Literature indicates a growing need to develop resources and enhance knowledge on cultural competence to assist health service providers to address the needs of people from diverse backgrounds. Yet, at what stage of training and/or practice are health professionals gaining access to this knowledge?

Consultation with local health services and tertiary education institutions revealed a gap in the knowledge of Asian mental health being taught in the curriculum of students undertaking training to become health professionals. Further research indicated a need and desire from tertiary institutions to have access to Asian mental health material for both enhancing students' learning, and promoting the concept of continuing education following graduation. As a result, an interactive, selfadministrative CD-rom comprising three modules: self-reflection; Asian philosophies; and clinical issues was compiled.
\end{abstract}

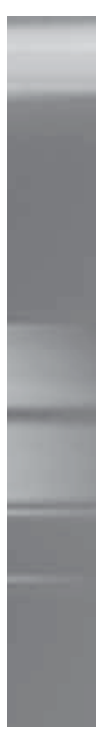

Early feedback indicates that the CD-rom has tremendous potential in terms of applicability to enhancing current curriculum and for teaching students skills such as accessing articles and web based resources, valuable for the purposes of continuing education. Continuing education with regards to cultural competence is not just for qualified health professionals but needs to be integrated as part of students training as health practitioners, in order to ensure health care providers are able to provide effective and culturally responsive services.

\section{Keywords}

Asian mental health; tertiary institutions; crosscultural training; graduate health professionals

\section{Introduction}

Continuing education is a mandatory requirement for health practitioners across all disciplines working within the New Zealand health system. An increasingly important aspect of continuing education is that of cultural competence. Currently, Asian people make up the fastest growing ethnic community in New Zealand, comprising $9.2 \%$ of the New Zealand population (Statistics 


\section{Enhancing cultural competence in mental health settings}

New Zealand, 2007). The Asian population comprises a diverse range of Asian communities and cultures with their unique worldviews. As the New Zealand population becomes more diverse, the need for the delivery of culturally competent and appropriate health care services is becoming paramount (Ho et al, 2002; Ministry of Health, 2004; Nursing Council of New Zealand, 2002). Cultural competence of the workforce is vital to ensure equity in access to appropriate and high quality care (Tse et al, 2005). Furthermore, literature indicates a growing need to develop resources on cultural competence to assist health service providers to address the needs of people from diverse backgrounds (Bhui et al, 2004; Tse et al, 2005; Tucker et al, 2003).

A key issue facing mental health services is a shortage of culturally competent trained workers to meet the needs of specific ethnic communities. According to the Strategic Principles for Workforce Development in New Zealand (Health Workforce Advisory Committee, 2006), New Zealand currently relies on overseas practitioners to 'ensure the availability of appropriate health services for specific ethnic communities' (Ibid, p8). Thus, access to a highly skilled, culturally competent and effective mental health workforce trained in New Zealand is a must to ensure ongoing delivery of culturally responsive services.

To date, the focus of educational programmes aimed at improving the appropriate skills, knowledge and cultural competence of the mental health workforce have centred on Mãori and Pacifica communities. Mãori are the indigenous people of New Zealand and have played a significant role in shaping today's society, while New Zealand has always been an important destination for migrants from the Pacific Islands. Currently, Polynesian people constitute the largest migrant community in this country. As such, policy documents addressing cultural responsiveness in the health sector have targeted these two communities (Ministry of Health, 1997; 2002a; 2002b; Nursing Council of New Zealand, 2002).

More recently, literature reviews have shed light on the competing needs of a wide variety of different ethnic groups (Ho et al, 2002; Tse et al, 2005). Thus, as part of developing a national strategy to improve mental health services, the Ministry of Health is committed to implementing a strategic approach to the Asian population's mental health workforce development (Ministry of Health, 2004). Health services that provide culturally competent care can prevent inappropriate advice, misdiagnosis, inappropriate treatments and inadequate interventions, therefore minimising the national health costs (Bedell et al, 2004; Bhui \& Bhugra, 1997; Bhui et al, 2003; Kirmayer, 2001). However, there have been few practical projects aimed at developing the workforce to meet the health and social services needs of the New Zealand Asian population.

In 2005, an educational training programme was implemented in four main cities across New Zealand to enhance the knowledge and capabilities of mental health clinicians working with Asian peoples (Nayar et al, 2007). Feedback from training participants highlighted the benefit and need for this type of training as health professionals in practice. The Beginning Phase of Asian Mental Health Training in Aotearoa, New Zealand (Tse et $a l$, 2006) report acknowledged that the project was only a starting point, a 'beginning phase', to implement such training nationwide, stating that 'further development is required in terms of ensuring this training is available to all mental health clinicians working with Asian people(s)' (Tse et al, 2006). Then again, should such training be withheld until clinicians are already practicing, or should this knowledge be made available at an earlier stage?

The purpose of continuing education in relation to cultural competence is to enhance mental health practitioners' knowledge about the profound and intrinsic impact of cultural factors on the prevention, presentation and treatment of mental health issues. In addition, education in cultural competence may facilitate more skilled interaction with Asian peoples in every aspect of training and psychotherapy (Falender \& Shafranske 2006). The mental health workforce would be better equipped to provide an enhanced culturally competent service if they had access to the relevant knowledge and skills training at earlier stage. The aim of this paper is to report the experience in designing and producing a training package on Asian mental health that potentially benefits students at both undergraduate studies and continuing education level.

\section{Continuing education at the undergraduate level}

Continuing education is primarily promoted in the workplace following graduation. However, it is the authors' contention that continuing education is a practice that needs to be ingrained in the work ethos of undergraduate 


\section{Enhancing cultural competence in mental health settings}

students training for careers as health professionals. The authors believe that it is important for students training as health professionals to grasp the concept of continuing education and learn the skills needed for this task prior to entering the workforce, in order that they may more effectively utilise continuing education in their career.

Consultation with health organisations within the mental health sector revealed a clear gap in the knowledge base of graduates entering the mental health workforce regarding Asian mental health needs. Also there was a need for students to continue their ongoing education after graduation in relation to specific service requirements such as service protocols and clinical tools. Thus, in order to increase awareness of Asian health needs, it was proposed that a training module be created so that students could access further learning as a way of continuing education. This could be done either with the guidance of a lecturer within the class setting or outside of the classroom on the students' own time.

\section{Clarifying the topic}

Prior to creating the training module, the authors undertook a scoping exercise with tertiary education institutions across the country. The purpose of this exercise was to clarify the need for Asian health training within the current curriculum; to create an opportunity to involve tertiary educational institutions across New Zealand; and ensure that undergraduate health students seeking a career in mental health complete their qualifications with some understanding of Asian mental health needs and issues. As part of the scoping exercise, three questions were asked.

1. What are the gaps in the current curriculum being delivered?

2. Was there a need for Asian mental health theory in the current curriculum?

3. If so, how best could this theory be delivered within the current curriculum and tertiary training environment?

The questions were emailed to 40 heads of department of undergraduate health programmes at 25 tertiary education providers. In total, 21 responses were received, representing a $52.5 \%$ return rate.

In response to question one, a resounding majority (18 of the 21 responses) stated that their respective curricular did not specifically cover Asian mental health, although Asians as a 'cultural group' were identified as part of a multicultural society within the curriculum. Regarding question two, 17 respondents indicated a need for specific training information/materials regarding Asian mental health. The most common reason for this response was the acknowledgement of the growing Asian communities within New Zealand society. Question three produced the most diverse answers, with a variety of delivery options proposed. These ranged from online resources, to workbooks, to lectures and workshops.

While the overall response to the need for Asian mental health to be covered in the curriculum was positive, there were reservations. Current pressures to address other ethnic groups such as Mãori (indigenous peoples of New Zealand) and Pacifica (cultural groups originating from the Pacific Islands eg. Tongan, Samoan), meant that it might not be possible to add another dimension, in already limited time. Some questioned whether at this stage an Asian mental health module was too specialised to go into the curriculum. It was suggested that not every student who undertakes a health qualification within the tertiary sector would necessarily go on to work in mental health, and work with Asians who are experiencing mental health difficulties. Given this limitation, it was felt that an Asian mental health module may be considered as an 'optional' rather than a mandatory part of the training. In addition, it was felt that perhaps an online resource of some kind (such as a workbook and related online activities) that students could access if it was an area of interest would be of benefit. In light of time constraints within the classroom and the nature of such specific knowledge, it could be argued that those students interested in Asian mental health would benefit from access to resources that both promote cultural competence and the skills required for continuing education in this area.

Based on the responses to the surveys, two key features necessary for the development of a learning package emerged. First, the package needed to be self-contained, in that it could be utilised by any learner without the need for an 'expert' to guide the process. Within New Zealand, there are few Asian mental health practitioners, meaning that guest speakers within tertiary education regions are not always accessible. Second, a learning package needed to lay the foundations of continuing education. Undergraduate students should be offered the opportunity to learn the basic, theoretical materials in a self-paced learning manner (thus meeting their own level of learning 


\section{Enhancing cultural competence in mental health settings}

need), with the intention that when they graduate, they learn specific details and skills as part of their ongoing education.

With this in mind, the project team developed a CD-rom, which contains a mixture of both interactive and didactic learning that allows for both formal teaching and self-learning of skills and knowledge, as well as participant reflection. Furthermore, activities are designed throughout the CD-rom to complement training sessions that involve the use of case studies (eg. first person account, personal experiences as a service user or caregiver), promoting reflective practice and clinical reasoning.

In addition to consultation with local health services and tertiary education institutions, the project team liaised with local mental health organisations to identify and consult with two Asian service users (one Chinese and one Korean) and one family member (also from the Chinese community) on the need for, and appropriateness of, the curriculum. Specific activities included the recruitment of a service user as a member of the advisory group and the involvement of two service users and a family member in recording their voices/perspectives on 'Working with Asian clients' as part of the CD-rom.

\section{Devising the curriculum}

Drawing on international literature, web-based teaching resources and materials collated for earlier training (Tse et al, 2006), an education module was created with the aim of providing psychological and sociological theory, and empirical information on three modules:

- self-reflection

- Asian philosophy

- mental health clinical issues.

Each module provides the student with:

- context and relevant considerations (eg. values, principles, target populations)

- session aims and objectives

- session content

- teaching and learning activities and corresponding objectives

- teaching resources/material

- key references.

The modules are designed with a high degree of flexibility so that teachers can either utilise the material during classroom sessions and/or the education programme can be loaded on the institutions' intranet for students to access as part of self-directed learning. Information on the CD-rom can be printed off and used within the class setting, such as the activity sheets, which may be beneficial to complete as part of a group exercise. In addition, students can be directed to different levels of readings depending on the type of training they are undergoing. In essence, the training modules seek to:

- raise students' self-awareness when engaging in crosscultural communication

- increase students' level of understanding about major Asian health-related philosophy and values by referring to relevant material and research studies

- highlight the key considerations when working with Asian mental health clients

- help students develop culturally appropriate skills in working with Asian clients (at entry level)

- introduce students to key literature on relevant topics.

Throughout the modules, an extensive reference list of pertinent readings is supplied, as well as links to credible web-based resources to encourage students to access further information, a skill necessary for undertaking continuing education. In this regard, the CD-rom aims not to provide all the answers to Asian mental health issues, but to provide students with an introduction to Asian mental health and establish the groundwork for continuing education in cultural competence as they embark on their careers as health professionals.

Module 1: Self-reflection. Self-awareness involves developing a basic understanding of life, society and the concept of culture, and knowing oneself as a cultural being, ie. world views, attitudes, values, assumptions, beliefs, behaviours, prejudices and preconceived ideas. Although self-awareness is a component of cultural competence, incorporation of an integrated awareness, understanding, and competence with one's own cultural or multidiverse background has been slow to appear in training environments (Falender \& Shafranske, 2006). Self-reflection can be an uncomfortable and threatening experience, while the process of self-discovery and acknowledging one's own cultural biases and prejudices may induce resistance, defensiveness and inhibition (Abreu, 2001). 


\section{Enhancing cultural competence in mental health settings}

This module incorporates continuing education activities through the use of two questionnaires regarding cultural self-assessment and knowing one's body language (Cutler, 2005a; 2005b). These questionnaires can be done outside the classroom with results brought back for discussion of learning with peers. The overall purpose of these questionnaires is to encourage students to reflect on their own understanding of culture.

Module 2: Asian philosophy. This module covers the Asian worldview - the way people see life and the world, cultures, values and cultural and religious practices as well as commonalities of values/beliefs across the Asian world, for example collectivism, spiritual beliefs, family and importance of education. Recent New Zealand demographics (Statistics New Zealand, 2007) indicate that attention to Asian cultural issues in mental health is a necessity, not an option. Thus, it is now imperative that the mental health workforce becomes culturally competent in Asian culture, its impact on mental health and its implications for treatment.

In Module 2, students are encouraged to work with peers to read through written material and discuss new understandings. An Asian philosophy quiz has been developed to ensure active reading and processing of material by asking general questions and testing retention of information on Asian philosophies. This quiz has been designed for students to complete outside of the class setting as and when they feel confident and can be a used as a tool for assessing the knowledge gained in a continuing education format or highlighting gaps in knowledge.

Module 3: Clinical issues. This module concentrates on specific issues or skills (introductory level) required when working with Asian people experiencing mental health problems. The clinical aspect of cultural competence for mental health workers is a highly specialised field. As such, it would be best delivered by practitioners in the field who have first hand experience of working with mental health patients and understand the specifics of the work (Tse et $a l, 2007)$. For this reason, practitioners present material on various clinical issues pertaining to Asian mental health. The introductory section entitled 'Considerations for working with Asian clients with mental illness' provides an overview of key factors to be considered in a therapeutic encounter between mental health practitioners and Asian clients. The remainder of the module consists of PowerPoint presentations with a voice-over by the expert clinician. The presentations cover a variety of topics and are designed for students to be able to work through either individually or as a group with peers.

\section{Responses to the CD-rom}

Evaluation of the programme consisted of two parts: student responses and teacher responses.

\section{Student perspectives}

On the front page of the CD-rom, an evaluation section was created. Students were encouraged to go online and complete this written evaluation once they covered the material. On completion of the evaluation, the form is automatically emailed back to the Centre for Asian Health Research and Evaluation (CAHRE, where the project is based) so that evaluations can be compiled and reviewed over time. As the funding for this project has ceased, it has been difficult to actively pursue student feedback; hence, responses to date have been limited. However, those received indicate that the CD-rom has been a useful tool in extending their knowledge about the mental health needs of Asian people living in New Zealand. Students have particularly benefited from listening to the PowerPoint presentations prepared by the Asian clinicians and consequently the case study discussions that evolved in the classroom. CAHRE is currently exploring ways to work with tertiary providers to collate student feedback and will require further feedback to fully evaluate the benefits of this CD-rom in the education of cultural competence for undergraduate students training to be health professionals.

\section{Teacher perspectives}

The second part to the evaluation was provided by tertiary institutions involved with implementing the education programme. Teachers were encouraged to stay in touch with the project team at CAHRE, via emails, to discuss how the educational programme has been implemented, what worked well, what could be improved etc. For the most part, teachers were impressed by the comprehensiveness of the programme and were readily able to identify slots in their current curricula where the information provided in this education programme could be successfully implemented. As per the students' feedback, teachers particularly liked the interactive aspect of the CD-rom and the presentations prepared by the 


\section{Enhancing cultural competence in mental health settings}

Asian clinicians. The involvement of Asian clinicians from multiple country of origins was seen as a strength of the training resources. Furthermore, some colleagues appreciated the flexibility of the learning modules, for example, some could be used in class, some could be accessed by students outside classrooms and students could be directed to different levels of readings depending on the type of training students are undergoing (eg. certificate vs. diploma level).

\section{Future development}

This project is a new initiative in the landscape of Asian mental health in Aotearoa, New Zealand. The project team believes that there is potential for this project to grow and become a recognised component of tertiary education for undergraduate students studying to become health professionals. Feedback collected from key stakeholders and educational institutions situated around the country indicated that as a learning resource, the CD-rom could be used by staff members within the university such as student health centre staff responsible for pastoral care for international students. Furthermore, some suggested that the CD-rom could in fact be used by practitioners as part of their continuing education. This feedback supports that continuing education should and can begin early on in the process of students becoming health professionals.

In the future, the project team envisage the need for liaison with prospective funders to introduce the training modules on Asian mental health to most of the mental health training institutions, and explore the need and ways of introducing the training modules to relevant professional groups, for example, New Zealand Psychological Association, and service providers such as primary health care groups. Introducing the learning modules to relevant educational and service providers nationwide in the long term will require continued examination and evaluation of the quality of delivery of the training modules and the impact of continuing education in the area of cultural competence.

The growth of the Asian population in Aotearoa, New Zealand creates a demand for health service providers to address the needs of people from diverse cultural backgrounds. As noted in the introduction, the needs of Mãori and Pacifica communities have, until recently, been the focus for continuing education in cultural competence. Introducing an Asian perspective does not necessarily mean competing for resources at the expense of the Mãori and Pacifica populations. Rather, there is the opportunity to develop a holistic, multicultural perspective that incorporates and develops what has worked well for Mãori and Pacifica people, while including dimensions specific to Asian populations, such as those outlined in Module 2 of the CD. To ensure that this happens successfully, mental health practitioners must be provided with knowledge about the influence of cultural factors on the prevention, presentation and treatment of mental health issues so that they are more skilled in interacting with peoples from diverse ethnic backgrounds.

Access to a highly skilled, culturally competent and effective mental health workforce can only be achieved with the participation of education and training organisations, among others. Health professionals are continuously learning from day one of their training, therefore continuing education should not be something that students think of only after graduation. It is argued here that the concept of continuing education should be implemented in current undergraduate curriculum training to ensure students are graduating with a level of cultural competence and an understanding of how to participate in continuing education once in practice. It is believed that this approach will strengthen students training as health professionals prior to commencing practice.

\section{Conclusion}

This project is a starting point to implementing cultural competence, with respect to Asian mental health, nationwide at undergraduate level. The training module described in this paper has been presented to tertiary education establishments across New Zealand. Positive feedback has acknowledged the need for this programme in today's education climate and that the resource will be an invaluable contribution to the development of a culturally competent health workforce. It is envisaged that in the coming years, this form of continuing education will be critical for not only individual mental health students, but also for ensuring the cultural competence of mental health services as a whole.

\section{Implications for workforce development}

- Continue to promote partnerships with tertiary training providers and other identified stakeholders to support delivery of training that facilitates the concept of continuing education at undergraduate level. 


\section{Enhancing cultural competence in mental health settings}

- Develop interactive resources that provide information on a topic and encourage students to seek further information, thus practicing skills required for continuing education.

- Utilise gaps in current undergraduate health programmes as they relate to workforce practice to identify topics for the development of continuing education programmes within tertiary institutions.

\section{Address for correspondence}

Dr Samson Tse

Associate Professor

Department of Social Work and Social Administration

Faculty of Social Sciences

The University of Hong Kong

Pokfulam Road

Hong Kong

Email: samsont@hku.hk

\section{References}

Abreu JM (2001) Theory and research on stereotypes and perceptual bias: a didactic resource for multicultural counseling trainers. The Counseling Psychologist 29 (4) 487-512.

Bedell SE, Graboys TB, Bedell E \& Lown B (2004) Words that harm, words that heal. Archives for International Medicine 164 (13) 1365-1368.

Bhui K \& Bhugra D (1997) Cross-cultural competencies in the psychiatric assessment. British Journal of Hospital Medicine $\mathbf{5 7}$ (10) 492-496.

Bhui K, McKenzie K \& Gill P (2004) Delivering mental health services for a diverse society. British Medical Journal 329 (7462) 363-364.

Bhui K, Mohamud S, Warfa N, Thomas C \& Stansfeld S (2003) Cultural adaptation of mental health measures: improving the quality of clinical practice and research. British Journal of Psychiatry 183 (3) 184-186.

Cutler J (2005a) The Cross-cultural Communication Trainer's Manual. Volume One: Designing cross-cultural training (pp269272). Aldershot: Gower.

Cutler J (2005b) The Cross-cultural Communication Trainer's Manual. Volume Two: Activities for cross-cultural training (pp105106). Aldershot: Gower.

Falender CA \& Shafranske EP (2006) Clinical Supervision: A competency-based approach. Washington, DC: American Psychological Association.
Health Workforce Advisory Committee (2006) Strategic Principles for Health Workforce Development in New Zealand. Wellington: Health Workforce Advisory Committee.

Ho E, Au S, Bedford C \& Cooper J (2002) Mental Health Issues for Asians in New Zealand: A literature review. Wellington: Mental Health Commission.

Kirmayer LJ (2001) Cultural variations in the clinical presentation of depression and anxiety: implications for diagnosis and treatment. Journal of Clinical Psychiatry 62 (13) 22-28.

Ministry of Health (1997) Moving Forward: The national mental health plan for more and better services. Wellington: Ministry of Health.

Ministry of Health (2002a) He Korowai Oranga: Maori health strategy. Wellington: Ministry of Health.

Ministry of Health (2002b) Whakatataka: Maori health action plan 2002-2005. Wellington: Ministry of Health.

Ministry of Health (2004) Improving Mental Health: The second national mental health and addiction plan 2005-2015: Consultation document. Wellington: Ministry of Health.

Nayar S, Tse S, Wong SW, Wali R, Thapliyal A \& Bhui K (2007) Working with Asian individuals: advancing the New Zealand mental health workforce. International Journal of Therapy and Rehabilitation 14 (5) 222-227.

Nursing Council of New Zealand (2002) Guidelines for Cultural Safety, the Treaty of Waitangi and Mãori Health in Nursing and Midwifery Education and Practice. Wellington: Nursing Council of New Zealand.

Statistics New Zealand (2007) Quick Stats about Culture and Identity: 2006 census. Available from: http://www.stats.govt. $\mathrm{nz} / \sim$ /media/Statistics/Publications/Census/2006\%20 Census\%20 reports/QuickStats\%20About\%20A\%20Subject/QuickStats\%20 About $\% 20$ Culte $\% 20$ and\%20Identity/qstats-about-culture-andidentity-2006-census.ashx (accessed July 2009).

Tse S, Bhui K, Thapliyal A, Choy N \& Bray Y (2005) Asian Mental Health Workforce Development Feasibility Project. Auckland: Health Research Council of New Zealand.

Tse S, Nayar S, Thapliyal A \& Bhui K (2006) Beginning Phase of Asian Mental Health Training in Aotearoa, New Zealand. Auckland: University of Auckland, Auckland UniServices Limited.

Tse S, Sobrun-Maharaj A \& Nayar S (2007) Asian Mental Health: Training and development for real skills. University of Auckland: Auckland UniServices Limited.

Tucker CM, Herman KC, Pedersen TR, Higley B, Montrichard M \& Ivery P (2003) Cultural sensitivity in physician-patient relationships: Perspectives of an ethnically diverse sample of low-income primary care patients. Medical Care 41 (7) 859-870. 
Copyright of Journal of Mental Health Training, Education \& Practice is the property of Pier Professional Ltd. and its content may not be copied or emailed to multiple sites or posted to a listserv without the copyright holder's express written permission. However, users may print. download, or email articles for individual use. 